\title{
Recognition of the Plasmodium spp. circumsporozoite protein by malaria inhibitory antibodies
}

\author{
I. Kucharska1 , E. Thai' ${ }^{2}$ A. Srivastava ${ }^{3}$, J. Rubinstein ${ }^{4}$, R. Pomès ${ }^{5}$, J. P. Julien ${ }^{6}$ \\ ${ }^{1}$ The Peter Gilgan Centre for Research and Learning, Hospital for Sick Children ${ }^{2}$ The Peter Gilgan \\ Centre for Research and Learning, Hospital for Sick Children, ${ }^{3}$ The Peter Gilgan Centre for \\ Research and Learning, Hospital for Sick Children, ${ }^{4}$ The Hospital for Sick Children, ${ }^{5}$ The Peter \\ Gilgan Centre for Research and Learning, Hospital for Sick Children, ${ }^{6}$ The Peter Gilgan Centre for \\ Research and Learning, Hospital for Sick Children \\ igakuch@gmail.com
}

Sporozoites of the malaria parasites from Plasmodium genus express a surface circumsporozoite protein (CSP) that contains an unusual central region consisting of multiple amino acids repeats. The central region of CSP is immunodominant and antibodies targeting the repeats can inhibit sporozoite infectivity. CSP is the antigenic component of the most advanced malaria vaccine to date - RTS,S/AS01. As RTS,S/AS01 offers only modest protection, it is important to gain a better molecular understanding of the humoral immune response against this Plasmodium antigen to guide the design of superior vaccines.

In this study, we have analyzed the structure and molecular interactions of potent monoclonal antibodies (mAbs) using molecular dynamics simulations, X-ray crystallography, and cryoEM. We discovered that inhibitory mAbs can accommodate subtle variances of the CSP repeating motifs, and, upon binding, induce structural ordering of CSP through homotypic interactions. Interestingly, some $\mathrm{mAb} / \mathrm{CSP}$ complexes formed spiral assemblies with a varied degree of compactness. We propose that homotypic antibody interactions leading to antibody clustering around CSP is a general immune mechanism of mammals to interact with repeating motifs on sporozoites across Plasmodium species. 